\title{
Invariant Texture Segmentation Via Circular Gabor Filters
}

\author{
Jainguo Zhang, Tieniu Tan, Li Ma \\ National Lab of Pattern Recognition, Institute of Automation, Chinese Academy of Sciences, \\ Beijing 100080, P.R. China, Email: \{jgzhang, tnt, lma\} @nlpr.ia.ac.cn
}

\begin{abstract}
In this paper, we focus on invariant texture segmentation, and propose a new method using circular Gabor filters (CGF) for rotation invariant texture segmentation. The traditional Gabor function is modified into a circular symmetric version. The rotation invariant texture features are achieved via the channel output of the CGF. A new scheme of the selection of Gabor parameters is also proposed for texture segmentation. Experiments show the efficacy of this method.
\end{abstract}

\section{Introduction}

Texture is a basic cue for human beings to recognize objects. Research on texture is a very important task in computer vision and its applications. It has been a very active topic in the past three decades.

There are several research focuses in the field of texture analysis, mainly including texture classification, texture segmentation, texture synthesis, shape from texture, etc [1]. Texture segmentation aims at localizing the boundaries between different textures on one textured image plane by classifying pixels based on their texture properties. In recent years, invariant texture analysis has been paid more and more attention due to its increasing importance. A great deal of wok has been done on this topic [2][3][14][15]. However most of the existing methods focus on invariant texture classification. Efforts on invariant texture segmentation are still very limited, though invariant texture segmentation is highly desirable [4].

Multichannel Gabor function has been recognized to be a very useful tool in computer vision and image processing, especially for texture analysis. The increasing research on Gabor analysis is motivated by biological findings. Numerous papers have been published on Gabor analysis since Gabor proposed the 1-D Gabor function [5][6][7][8][9][10][12][16]. Researchers have agreed that Gabor-like linear spatial filtering plays a crucial role in the function of mammalian biological vision systems, particularly with regard to textures.

In this paper, we discuss invariant texture segmentation based on mutichannel analysis. The traditional Gabor filter is modified into a circular symmetric version. A very important property of this new version is that it is rotation invariant. Texture images are decomposed into several channel outputs. Texture features are computed from each channel output. Thus the feature space of each pixel is constructed. We also study the selection of Gabor parameters that is a very important problem. A new selection scheme is proposed for texture segmentation. $\mathrm{K}$-means clustering algorithm is employed for pixel classification. Experimental results have demonstrated the performance of this method.

\section{Circular Gabor filters and rotation invariance}

Gabor filter has been already demonstrated to be a powerful tool in texture analysis. Traditional Gabor function (TGF) is a Gaussian function modulated by an oriented complex sinusoidal signal. It is mathematically given by

$$
G(x, y)=g(x, y) \exp (2 \pi i f(x \cos (\theta)+y \sin (\theta)))(1)
$$

where $g(x, y)=\frac{1}{\mathbf{2} \pi \sigma^{2}} \exp \left(-\left(x^{2}+y^{2}\right) / \mathbf{2} \sigma^{2}\right)$ (here we assume that $g(x, y)$ is isotropic). The parameter $f$ and $\theta$ represent the frequency and the orientation of the sinusoidal signal respectively. $g(x, y)$ is the Gaussian function with scale parameter $\sigma . f, \theta$, and $\sigma$ constitute the parameter space of Gabor filters where $\theta$ lies in the interval $\left[0^{0}, 360^{\circ}\right]$. An example of the spatial response of a traditional Gabor filter is shown in Fig 1. Gabor filters have many advantages over the Fourier transform. It can achieve the optimal location in both the spatial and frequency domain.

Gabor filters are very useful in the detection of texture direction. This is a main advantage of traditional Gabor filters. But in rotation invariant texture analysis, the orientation of texture becomes less important. Thus traditional Gabor filters are less suitable for this topic. The sinusoidal grating of the TGF varies in one direction. If the sinusoid varies in all orientations, it is circular symmetric. This results in a new version of Gabor filters--circular Gabor filters (CGF). The circular Gabor filter is defined as follows:

$$
G(x, y)=g(x, y) \exp \left(2 \pi i F\left(\sqrt{x^{2}+y^{2}}\right)\right)
$$




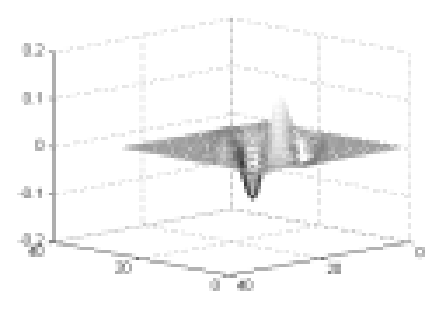

\section{Figure 1. An Example of a traditional Gabor function shown in the spatial domain}

where $F$ is the central frequency of a circular Gabor filter. The properties of the circular Gabor filters can be more explicit in their frequency domain. The Fourier representation of the circular Gabor filter is as follows:

$$
\begin{aligned}
& F(u, v)=\frac{\sqrt{2 \pi}}{2} \alpha \exp \left(-\frac{\left(\sqrt{u^{2}+v^{2}}-F\right)^{2}}{2 \alpha^{2}}\right) \\
& \text { where } \alpha=\frac{1}{2 \pi \sigma} . \text { Fig. } 2 \text { and Fig. } 3 \text { show examples }
\end{aligned}
$$

of spatial response and Fourier representation of a

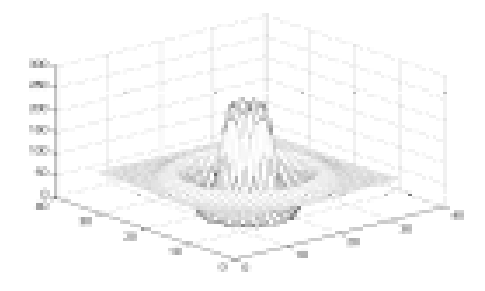

Figure 3. An example of a circular Gabor function shown in the spatial domain

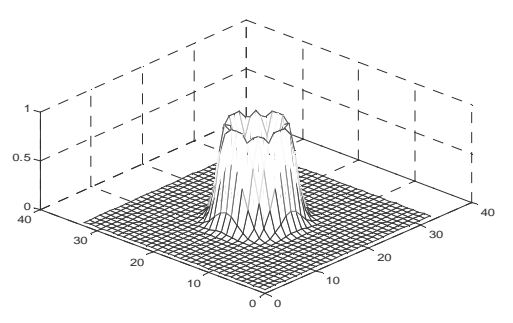

\section{Figure 2. An example of a circular Gabor} function shown in the spectrum domain

circular Gabor filter. In fact, in Gabor based texture analysis, texture property of each pixel is provided by the projection of the textured surface $I(x, y)$ onto a complex Gabor wavelets. That is (here we use circular Gabor filter) :

$$
P=\iint I(x, y) g(x, y) \exp \left(i 2 \pi F \sqrt{x^{2}+y^{2}}\right) d x d y
$$

Consider a rotation of texture image $I\left(x^{\prime}, y^{\prime}\right)$ by an amount of $\Delta \theta$. The projection of $I\left(x^{\prime}, y^{\prime}\right)$ becomes:

$$
P^{\prime}=\iint I\left(x^{\prime}, y^{\prime}\right) g(x, y) \exp \left(i 2 \pi F \sqrt{x^{2}+y^{2}}\right) d x d y
$$

where $\left[\begin{array}{l}x \\ y\end{array}\right]=\left[\begin{array}{cc}\cos \Delta \theta & \sin \Delta \theta \\ -\sin \Delta \theta & \cos \Delta \theta\end{array}\right]\left[\begin{array}{l}x^{\prime} \\ y^{\prime}\end{array}\right]$. We have $d x d y=d x^{\prime} d y^{\prime}$ and $x^{2}+y^{2}=x^{\prime 2}+y^{\prime 2}$. Thus the above equation can be represented as follows

$$
P^{\prime}=\iint I\left(x^{\prime}, y^{\prime}\right) g\left(x^{\prime}, y^{\prime}\right) \exp \left(i 2 \pi F \sqrt{x^{\prime 2}+y^{\prime 2}}\right) d x^{\prime} d y^{\prime}(6)
$$

From Equation 4 and 6 , we can obtain that $P=P^{\prime}$. That is we have proved that when an image is rotated, its projection onto a circular Gabor wavelet remains the same. This property provides the rotation invariance.

\section{Parameter selection}

The selection of Gabor parameters has been a long research focus in the field of Gabor based image processing. A great deal of work (including physiological research and theoretical algorithms) has been done on this important issue [7][9][17][18]. Many methods have been proposed to reduce the degrees of freedom of Gabor filters based on neurophysiological findings [17]. One important finding is that the spatial-frequency bandwidth of the response of the human visual cortical cells ranges from 0.5 to 2.5 octaves. For a circular Gabor filter, $F$ and $\sigma$ satisfy the following condition that is controlled by the bandwidth $B$ of the Gabor filter [12]

$$
\begin{aligned}
& F \sigma=\lambda \frac{\left(2^{B}+1\right)}{\left(2^{B}-1\right)} \\
& \text { where } \lambda=\frac{\sqrt{2 \ln 2}}{2 \pi}, \quad B=\log _{2}\left(\frac{f_{1}}{f_{2}}\right) \quad \text { with }
\end{aligned}
$$

$f_{1}$ and $f_{2}$ the frequencies corresponding to the half peak magnitude of circular Gabor Fourier response. In this paper we set the value of the bandwidth of the Gabor filter 1 octave. Thus we have $F \sigma=3 \lambda$. For a texture image of size $\mathrm{NxN}$, the frequency $F$ is often selected as follows (normalized by $\mathrm{N})[12][13][19]$ :

$$
\sqrt{2}\{1 \quad 2,4, \quad 8, \quad 16,
$$

This choice implies finer frequency resolutions in lower frequencies than in higher frequencies, i.e. it emphasizes the lower frequencies. This choice may result in very good texture representation. However, good reconstruction does not mean good segmentation (see [20] for more details). Furthermore Chang and Kuo [19] have already pointed out that texture features are often prevalent in the intermediate frequency bands. From this point we propose another frequency selection scheme, which emphasizes the intermediate frequency band. The new choice is as follows (Note that the frequency can be normalized into [-0.5 0.5$]$ by the image size $\mathrm{N}$, but due to the symmetry of Fourier spectrum, only half part [ $\left[\begin{array}{ll}0 & 0.5\end{array}\right]$ is considered here.): 


$$
\begin{array}{ll}
F_{H}=0.25+2^{(i-0.5)} / N & 0.25=<F_{H}<0.5 \\
F_{L}=0.25-2^{(i-0.5)} / N & 0<F_{L}<0.25 \\
i=1,2, \cdots \quad \log _{2}^{(N / 8)} &
\end{array}
$$

The comparison between the traditional frequency selection scheme and our new scheme is illustrated in Fig. 4. It can be seen that the curve of the new selection is much flatter in the intermediate frequency band than the traditional one. This indicates that our choice of central frequency does have finer resolutions in the intermediate frequency (around 0.25).

Our choice of Gabor filters does not consider the very high frequency band of image (0.4-0.5). This can also be seen from Fig. 4. That is the Fourier response of the Gabor filter does not cover corners of frequency domain. This is due to the following reasons: (1) most of the spectral energy of natural image often centres at low frequency. There is very little energy at high frequencies [1]. (2) Furthermore, it can keep the computational efficiency. It is obvious that convolution of an image in their spatial domain will inevitably result in a high computational cost. To avoid this, we implement the convolution in the frequency domain.

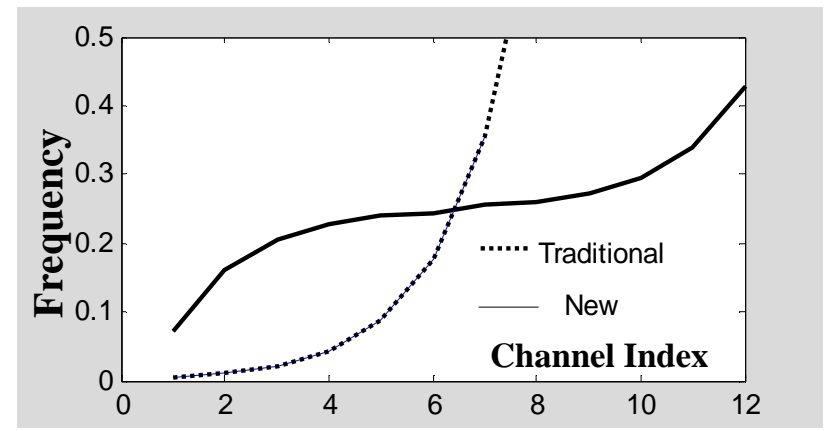

Figure 4. Two selections of Gabor channel center frequency sampling

\section{Texture segmentation}

The output of the Gabor channel represents texture information at different spatial frequency. For texture segmentation, we should define the texture measurements on the filtered image. We calculate the texture features from the corresponding filtered image as follows:

$\phi(x, y)=r^{2}(x, y) \otimes m(x, y)$

where $r^{2}(x, y)$ is the energy of the filtered image of each channel, $\otimes$ represents convolution and $m(x, y)$ is the mask used to localize the region to obtain texture measurement. Mask windows of large size tend to produce the more reliable but coarse texture measurements. They are not suitable for texture boundary localization. Mask windows of small size can produce finer texture measurements, but of less robustness. In our experiment, we use the Gaussian window to estimate the local texture energy. The window size is determined by its standard deviation $\sigma_{s}$. We choose $\sigma_{s}=2 \sigma$ (here $\sigma$ is the scale parameter of a circular Gabor Filter. See Equation (7)) which has already been found suitable for texture segmentation [4][12][[20]. Suppose that for a given texture image, a series of Gabor channel output can be represented as $r_{i}(x, y) \quad i=1,2,3 \ldots \mathrm{T}$. The feature vector of each pixel is constructed by $\left\{\phi_{i}(x, y) \quad i=1,2,3 . . . \mathbf{T}\right\}$. The dimensionality of the feature space is the same as the number of Gabor channels T. Thus each texture class forms a relatively compact and isolated region in the feature space. Pattern clustering algorithm is required to identify these regions. In our scheme, we employ the k-means clustering algorithm. It iterates until the center of each texture class does not change.

\section{Experimental results}

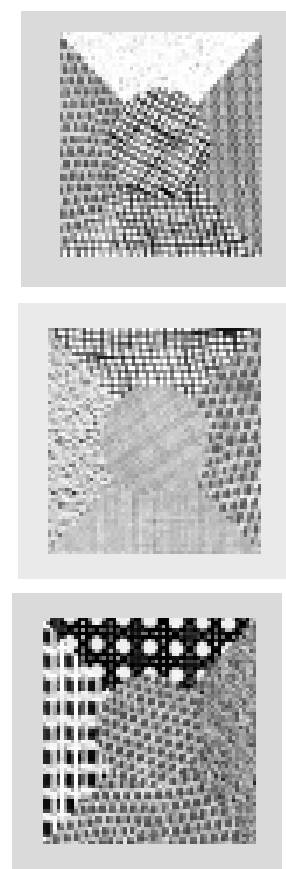

( a )
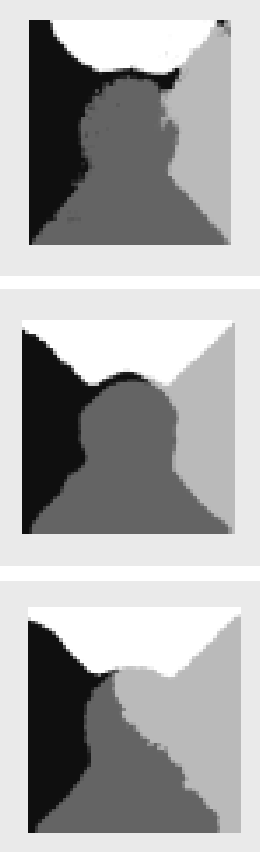

( b )
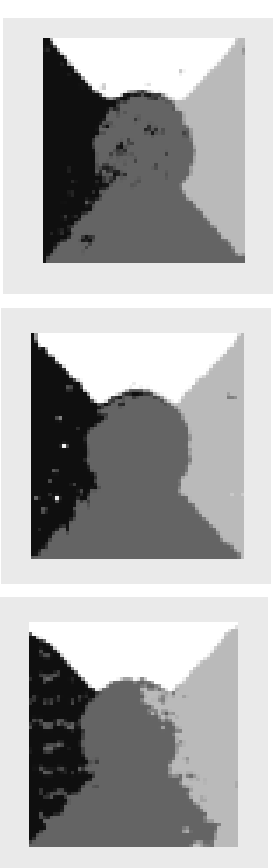

( c )
Figure 5. Rotation invariant texture segmentation results. ( a ) Three $256 \times 256$ images containing four natural Brodatz textures. ( b ) rotation invariant texture segmentation using CGF based on the traditional scheme of frequency selection. ( c ) rotation invariant texture segmentation using CGF based on the new scheme of frequency selection

In order to test the performance of our algorithm, we apply it to several test images for rotation invariant texture segmentation. These test images are constructed 
by sticking several natural textures from the Brodatz album and their randomly rotated versions. These images include both random and regular textures. Each test image is of size $256 \times 256$. We perform our experiments in two cases. In the first case, the center frequency of each CGF channel is selected using traditional scheme as discussed in Section 3. In the second case the center frequency of each CGF channel is determined according to Equation 8. The bandwidth of each channel is kept at 1 octave in both of the above cases. We use the misclassification rate to evaluate the segmentation performance in the two cases. In the first case, the error rate for each test image is $7.69 \%, 4.33 \%$, and $14.22 \%$, from top down in Fig.5. In the second case the corresponding error rate is $5.26 \%, 3.02 \%$, and $6.70 \%$. The segmentation results are shown in Fig.5. From this figure and the experimental data, two valuable observations can be made: (1) the regions containing one texture and its rotated versions have been well grouped into one region. This indicates that our proposed method based on CGF has good performance in rotation invariant texture segmentation. (2) Compared with the traditional selection scheme, our new scheme of central frequency selection has better segmentation results (in terms of better pixel classification accuracy). Apparently the segmentation results in Fig. 5 may be further improved by removing small holes (e.g. by means of morphological filtering) but this is not the main focus of the current paper.

\section{Conclusions}

In this paper, we have presented a new rotation invariant texture segmentation method based on circular Gabor filters. The projection of images onto circular Gabor filters is rotation invariant in nature. We have discussed the problem of filter parameter selection. A new scheme of central frequency selection is also proposed. Our experiments show that this scheme demonstrates good performance in rotation invariant texture segmentation. Further research focus may be on testing the efficacy of this method on noisy images. Moreover, how to reduce the number of channels without compromising the segmentation results is an interesting problem to be investigated.

\section{Acknowledgement}

This work is funded by research grants from the NSFC (Grant No. 69825105 and 69790080) and the Chinese Academy of Sciences.

\section{Reference}

[1] M. Tuceryan and A.K. Jain, Texture Analysis, in Handbook of Pattern Recognition and Computer Vision (C. H. Chen, et. al., Eds.), 1993, pp.235-276.
[2] J.G. Zhang, T. Tan, Brief Review of Invariant Texture Analysis Methods. Pattern Recognition, Vol. 35/3, pp. 735-747, 2002.

[3] T.N. Tan, Geometric Transform invariant Texture Analysis, SPIE, Vol. 2488, (1995) pp475-485.

[4] R. Chellappa and R.L. Kashyap and B.S. Manjunath, Model Based Texture Segmentation and Classification, in Handbook of Pattern Recognition and Computer Vision (C. H. Chen, et. al., Eds) (1993), pp.277-310

[5] D. Gabor, Theory of Communication, J. Inst. Elect. Eng London, 93 (III), 429-457, 1946.

[6] J. P. Havlicek, A. Bovik, D. Chen, AM-PM Image Modeling and Gabor Analysis, Visual Information Representation, Communication, and Image Processing (C. H.Wen, Y.Q. Zhang, Eds.), New York, Marcel Dekker, 1999, pp.343-386.

[7] D. J. Field, Relation Between the Statistics of Natural Images and the Response Properties of Cortical Cell, $J$. Opt. Soc. Am. A. 4, 2379-2394 (1987).

[8] A. C. Bovik, N. Gopal, T. Emmoth, and A. Restrepo, Localized Measurement of Emergent Image Frequency by Gabor Wavelets", IEEE Trans. on Inf. Theory, 38, 2, 691-712(1992).

[9] O. Nestares, R. Navarro, T. Portilla, and A. Tabernero, Efficient Spatial-domain Implementation of Multiscale Image Representation Based on Gabor Functions. J. Electronic Imaging, 7, 166-173 (1998).

[10] J.Daugman, Uncertainty Relation for Resolution in Space Spatial Frequency and Orientation Optimised by Two Dimensional Visual Cortical Filter, J. Opt. Soc. Am. Vol. 2, No. 7, pp. 1160-1169, 1985.

[11] J. Daugman, C. Downing, Demodulation, Prediction, Coding, and Spatial Vision, Journal of the Optical of Society of America, A 129(4) (1995) pp. 641-660.

[12] A.K. Jain and F. Farrokhnia, Unsupervised Texture Segmentation Using Gabor Filters, Pattern Recognition, Vol.24, No.12 (1991), pp1167-1186

[13] Tai Sing Lee, Image Representation Using 2D Gabor wavelets, IEEE Trans. Pattern Analysis and Machine Intelligence, Vol. 18, No. 10, Oct. 1996, pp.1-13.

[14] S. Fountain and T. Tan, Extraction of Noise Robust Invariant Texture Features via Multichannel Filtering, Proceedings of ICIP '97, Vol. 3 (1997), pp197-200.

[15] T.N. Tan, Rotation Invariant Texture Features and Their Use in Automatic Script Identification IEEE Trans. Pattern Analysis and Machine Intelligence Vol.20, No.7, July 1998, pp. 751-756.

[16] M. Turner, Texture Discrimination by Gabor Functions, Biol. Cyber, 55, pp.71-82, (1986).

[17] J. Portilla, R. navarro, O. nestares, and A. Tabernero, Texture Synthesis-by-Analysis Based on a Mutiscale Early Vision Model, Optical Engineering, 35, pp. 2403-2417 (1996).

[18] D. J. Heeger, Model for Extraction Image Flow, J. Opt. Soc. Am. A 4, 1455-1471 (1987).

[19] T.Chang and C. -C. J. Kuo, Texture Analysis and Classification with Tree-structured Wavelet Transform. IEEE Trans. Image Processing, 2 (4) pp.429-441, 1993.

[20] T. Randen, J. H. Husoy, Filtering for Texture Classification: a Comparative Study. IEEE Trans. on PAMI, No.4, pp.291-310, 1999. 